\title{
Why Is the Chinese Curriculum Difficult for Immigrants' Children from Southeast Asia
}

\author{
Chiu-Jung Chen ${ }^{1, *}$ \\ ${ }^{1}$ Department of E-Learning, Design and Management, National Chia-yi University, Taiwan \\ *Correspondence: Department of E-Learning, Design and Management, National Chia-yi University, Taiwan. E-mail: \\ alexch50@gmail.com
}

Received: April 4, 2016

Accepted: May 12, 2016 Online Published: July 18, 2016

doi:10.5430/jct.v5n2p1

URL: http://dx.doi.org/10.5430/jct.v5n2p1

\begin{abstract}
The purpose of this study is to explore the difficulties of immigrants' children from Southeastern Asia on learning Chinese phonetic symbols and provide further instructors a reference while educating them in early childhood. The participants are native Taiwanese children $(N=40)$ and immigrant children from Southeastern Asia $(N=34)$ at the first grade in ten elementary schools in Taiwan. The result demonstrates that the first grade immigrants' children easily made errors on recognizing consonants and vowels in the part of "Chinese phonetic symbol test". For pronunciation, the immigrant children got a high percentage of making mistakes on falling-rising sounds.
\end{abstract}

Keywords: phonetic difficulties; immigrants'children; Southeast Asia; Chinese curriculum

\section{Introduction}

Language is the tool that human use it to communicate and express their feelings. People organize, connect, integrate and convey massages though language skills, and also apply it into knowledge learning and problem solving (Bernstein \& Tiegerman-Farber, 2002). Reading is not only an effective way to get knowledge and message, but also the essential tool and medium for learning other subjects. Children, who are great at reading and have skill and resource to broaden their potential, will get good grade on school work (Ho, Chan, Chung, et al., 2007). On the other hand, children, who are not good at reading, will lead to fail to acquire knowledge successfully and even cause the obsession of feelings and behavior (Pumfrey \& Elliott, 1990 ; Stanovich \& Stanovich, 2006). Therefore, it is an important education topic on how to cultivate children's reading ability.

Tan (1998) thought that the difference of the language communication styles will influence children' adjustment of school. The language development of minority students will be poorer or their communication style will differ from the majority students. They are situated in disadvantageous status of language learning activities and have more learning besetment. With technology development and frequency of international interchange, there have emerged a lot of marriage immigration from south-east since Taiwan's eighties which made another immigration climax after 1950's Mainlander immigration. It is different from Taiwan's past immigration structure. No matter in races, their language cultures or life customs are quite different from Taiwanese (Chen, 2003). According to the information from the Department of Household Registration adding up until 2014, there are about one-third of the first grade students in elementary school born by foreign spouse. The highest is from Vitamin $(67.14 \%)$, the second one is from Indonesia (14.74\%), and the third one is from Thailand (5.87\%) (Department of Statistics, 2014).

The difficulties of immigrants' children from Southeastern Asia on learning Chinese phonetic symbols have not been explored. Thus, in this study, we focus on the problem of children born by foreign spouse in acquiring Taiwan's phonetic symbols.

\section{Literature Review}

Reading is the comprehensive ability of language, an embedded ability in a short time; in other words, reading ability is the understanding of words, sentences, paragraphs, and articles (Lin, 1997). When reading, it not only needs the ability of cognition but also needs skills, such as grab the key words, and skip over the barriers in order to improve 
the reading benefit. Like Goodman (1992) said, readers will use the clues from context, the background knowledge, and linguistic knowledge to get the idea of the article when reading. Reading is absolutely a vital tool in daily life, especially in the society that the technical product changes with each new day. Knowledge acquisition is no longer merely taught by professors. Learners have to find data, read, think, convert by themselves. Thus, they can convert outside information into his own property. Therefore, if readers read voluntarily and have frequent reading behavior, their knowledge and mind will develop much better (Zeng, 2003).

Phonological awareness has been regarded as the main defect to those reading disabled children (Kuder, 1997). In Chinese, there are three main parts, initial consonant, rhyme, and tone. Rhyme can be separated into three parts, including prevocalic glide, main vowel, and terminal sound. Therefore, the most completing syllable structures of pronunciations in Chinese characters are initial consonant, prevocalic glide, main vowel, terminal sound, and tone (Zhong, 2004). Chinese phonetic symbols are the symbol of pronunciation of Chinese characters. By learning Chinese phonetic symbols, it can combine pronunciation and Chinese characters. Meanwhile, via pronunciation, there will be a bridge between lexical form and meaning. Thus, learning Chinese phonetic symbols help children learn Chinese characters and improve reading ability (Huang, 1997).

According to the studies (Hu, 2001; Zhang, 1997), the easier part in initial consonant is bilabial, the most difficult part is the front and back sounds pronounced by the tip of the tongue. About the rhvme, the sounds [a] Y, [i] and [u] $X$ are the easiest sounds to learn; the sounds with rhyme "ê,", [ei] $\mathcal{Y},[\mathrm{o}] \mathcal{C}$ and [ou] $又$ are the most difficult. When it comes to spelling. the variation varies because of the use of materials, but words that contain the rhyme [a] Y, [i] - , and [u]X," and bilabial sounds are easier to spell. However, the spelling of fricative, affricative, and the sounds with rhyme are not easier to spell.

As for tone, first and the forth tone are the easiest to learn; second and the third tone are difficult to learn. But there are two studies show that the fires tone is the most difficult sound to recognize. From the researches above, the studies show that the difficulty of Chinese phonetic symbols match to general phonetic development. Bilabial sounds are the earliest, and then the fricative and affricative, the sounds with rhyme are hard to pronounce (Bernthal \& Bankson, 2004). Hong (1993) found that the ability of phonological awareness of reading disabled children is worse than normal children who are at the same age. Zeng (1993) took bilingual children in Singapore as subjects in order to discuss the relation between phonological awareness and scores. The result is that the child who is an English speaker, whose phonological awareness has something to do with Chinese and English scores; as a Chinese speaker, whose phonological awareness has nothing to do with reading scores.

Even though many studies discussed how and why elementary students in Taiwan have the difficulty in learning Chinese phonetic symbols, little research investigates the difficulties of immigrants' children from Southeastern Asia on learning Chinese phonetic symbols. Thus, in this study, we focus on the problem of children born by foreign spouse in acquiring Taiwan's phonetic symbols.

\section{Methodology}

\subsection{Subject}

The main subjects of this study are the first grade elementary school students, whose mothers are from Southeast Asia $(N=34)$, while others are Taiwanese $(N=40)$. The immigrant children's level of intelligence must be within the normal range, and must have live with immigrant mother and father who is Taiwanese. Researcher chose ten medium-size elementary schools in Taiwan to ask volunteer to participate this study.

As a result, there were 34 immigrants' children, whose mothers are from Southeast Asia, such as Vietnam and Indonesia (see Table 1). Among these, there are 16 boys and 18 girls. Mothers who are Vietnamese are in the majority $(\mathrm{N}=6), 6$ students from Indonesia; six from Philippines; four from Cambodia; six from Malaysia; two from Myanmar, Thailand, and Macao respectively.

Table 1. Distribution of Sample Participants

\begin{tabular}{|c|c|c|c|c|c|c|c|c|c|c|}
\hline \multirow[b]{2}{*}{ Total } & \multicolumn{2}{|c|}{ Gender } & \multicolumn{8}{|c|}{ Mother's Nationality } \\
\hline & Boys & Girls & VNM & IDN & PHL & KHM & MYS & MMR & THA & MAC \\
\hline 34 & 16 & 18 & 6 & 6 & 6 & 4 & 6 & 2 & 2 & 2 \\
\hline
\end{tabular}

Note: VNM: Vietnam, IDN: Indonesia, PHL: Philippines, KHM: Cambodia; MYS: Malaysia; MMR: Myanmar, THA: Thailand, MAC: Macao 


\subsection{Instrument}

Chinese phonetic symbol test sheet is a tool to estimate immigrant children's spelling of Chinese phonetic symbols. The test applies to the elementary students at first grade. They are just finished the Mandarin text book I. The test parts into two sections: listening and writing, identification and reading.

(1) Listening and writing test: There are 16 questions, including listening and writing down the phonetic symbols (e.g., ê, er [ə] 儿), initial consonants and rhymes will be shown alternately. Eight items for single sounds (e.g., [tsan]), other eight items about words and phrases (e.g., "pai", "shou"). This part takes about ten minutes. The reliability part of the part of identification and reading is .96. The following is the guideline to do the test:

Attention, please. Now the test begins. The teacher will say each topic three times. All of you have to concentrate on it. And then write down the correct answer in the blank. The teacher will say the topic with direct-spelling.

(2) Identification and reading test: There are 35 questions about identification of phonetic symbols, initial consonants and rhymes will be shown alternately. Twenty questions of this part about spelling and reading short essay, including single phonetic symbols, double-spelling sounds, triple-spelling sounds, and the change of tone. The reliability of this part is .95 . This test is an excellent stability.

\section{Results}

\subsection{The Mistakes That Students Have on Listening and Pronunciation}

In the phonetic symbols of Chinese characters, CG students have few mistakes on $\mathrm{t}, \mathrm{s}, \mathrm{n}, \mathrm{r},(0.9 \%-3.31 \%)$ but have many mistakes on s, c $(5.12 \%, 14.45 \%)$. EG students have no mistakes on $\mathrm{CCt}, \mathrm{n}, \mathrm{r}$ but have many mistakes on $\mathrm{s}, \mathrm{f}$ (11.11\%) and the mistakes on $\mathrm{s}$ are up to $22.22 \%$. Having a full view of the phonetic symbols of Chinese characters, regardless of their identities, the error rate on $t, n, r$ is not high but new immigrant students' rate of making wrong conjecture on s, f, c students is higher than common students. Compare with the phonetic symbols of Chinese characters, the expression of common students on the prosodic symbols of ê, s, ei, an, ei is not well and the rate of wrong answer of "eng" is $10.54 \%$, the highest error rate. New immigrant students' expression of the prosodic symbols is better than the phonetic symbols of Chinese characters, and they have no mistakes (see attachment 2, form 1).

(2) Errors in phonetic transcription: That student has difficulties in phonetic transcription will related to what we refer above. Also, there are some situations caused by the wrong way to link up the phonetic symbols. Then we are investigating what phonetic symbols combine will easily make these two problems, and the types between every group. Native Taiwanese children do a good job on link up the phonetic symbols, they seldom make mistakes, only in "wo" and "o" combined, they are easy pronouncing incorrectly for the wrong way, and then the probability of being wrong is $7.53 \%$. Immigrant children are inferior in linking up the phonetic symbols, the probability of being wrong on "wo", " xue" are orderly $11.11 \%$ and $22.22 \%$, it is worthy investigate the effect of their mother language (detailed sample in appendix two chart one).

For different types on pronouncing, native Taiwanese children are easily confused with "chao mian" and "zhang yu" in the second part- linking words, but Immigrant children are superior in this part (detailed sample in appendix two chart two). For the third part, probability of being wrong for native Taiwanese children on "re nao"," dou fu", guan deng", shai tai yang", " jin tian" is up to $10 \%$, among them " jin tian" is up to $34.94 \%$, which not only because it's difficult distinguish " en eñ ", but part of students choose "sunny day" is a nice weather, and this show that the failed question make students judge it incorrectly. Immigrant children are superior in this part, and never make mistakes (detailed sample in appendix two chart one).

Although immigrant children are superior in the second and third part, they are inferior to native Taiwanese children in the forth part- circle the correct answer and the fifth part- reading pictures then fill up the phonetic symbols. native Taiwanese children in the forth part- circle the correct answer have higher probability of being wrong on "kuan da" and "gan kuai", which reveal that "kuan guan" and "gan gang" are hard to distinguish; the probability of being wrong of immigrant children on " er tong", " yan lei", " cai hong", "gan kuai", " zhang gao" is up to $10 \%$, which reveals that " er e"," lei lie", "cai cai", " gan gang" and " chang zu" are hard to distinguish, and among them, " gan gang" and " chang zu" orderly are up to $33.33 \%$ and $22.22 \%$ (detailed sample in appendix two chart one). For the fifth part - reading pictures then fill up the phonetic symbols, probability of being wrong for native Taiwanese children only on " mian yang" is up to $10 \%$, but the reason why many people write "xiao yang", it's because they have different cognition from the correct answer; probability of being wrong for immigrant children on " peng you", "xiao gou", " mian yang", " cao mei", "song shu", " dian nao"and " pu tao" are up to $10 \%$, from that it is obvious to 
see that it's hard to distinguish $\mathrm{k}, \mathrm{g}$, ch, c, s, sh from en, eng (detailed sample in appendix two chart five).

(3) Errors in tone: performance of tone are related to whether phonetic symbols combine and pronounce is correct or not. What follows will investigate the incorrect situation and condition in every group. probability of being wrong for native Taiwanese children on "qiao" is the highest $11.74 \%$, only for tone, the falling-rising tone are higher than other tones. Probability of being wrong for immigrant children also on "qiao" is the highest $11.11 \%$, "xue" this sound will influence the judge of tone for the wrong pronounce of combined rhyme itself, the judge of tone for the falling-rising tone is also high.

From what refers above, immigrant children are easily judge wrong tone on $\mathrm{f}, \mathrm{h}, \mathrm{g}, \mathrm{k}, \mathrm{s}, \mathrm{sh}, \mathrm{ch}, \mathrm{c}, \mathrm{r}, \mathrm{l}$, en, eng, an, ang. There are more difficult on "wo", "xue" and" lei lie" these combined rhyme. Only for tone, the falling-rising tones is higher than other tones. Chinese phonetic symbol is the basic of Chinese education. Through learning Chinese phonetic symbol, students can pronounce correctly when learning to speak Chinese, and also in terms of them to direct students to realize and write symbols and help them reading, knowing words, speaking and writing composition. The most importance is that students can rely on correct and fluent language in daily life, and amply express feelings communicate thoughts and emotions between people; therefore, Chinese phonetic symbol is the basic of Chinese education, and also affect directly on other subjects. Then, it can't ignore the importance of Chinese phonetic symbol education.

Nowadays the Chinese phonetic symbol education in elementary school emphasize on daily lives and interesting. It starts from sentence then read the words, tones and Chinese phonetic symbol carefully. Although it emphasize on start from students' former experience, it also has complexity and difficulty on learning sentence and spelling at the same time when students are not familiar with symbols. Remedy teaching will make up for differences between every student in accordance with their demands and weakness, then try to help them catch up with the schedule and reach the purpose before they are contact with more difficult course. Therefore, it is necessary to carry out remedy teaching which contain its strong point.

\section{Conclusion}

In terms of Chinese phonetic alphabet, the reason of causing the tendency for foreign bride's children to have a comparatively bad performance than Taiwanese children is mainly due to the difficulty of distinguishing the pronunciation of similar Chinese phonetic alphabet. To learn Chinese phonetic alphabets is the primary goal for our national education, and some scholars had found that there is a strong relationship between recognizing Chinese characters and Chinese phonetic alphabets. At the same time, the connection is high among spelling, Chinese phonetic alphabet, and recognizing Chinese characters. From the evidence above, we could see that learning Chinese phonetic alphabets do help students to know more characters. As a beginner, students in the first grade of elementary school have to learn Chinese phonetic alphabets for ten weeks.

\subsection{Frequent Mistakes}

From the compile statistics, the easiest sound for foreign bride's children to pronounce include " $b, \mathrm{p}, \mathrm{I}$, wu, a" but the hardest sounds for them are " $\mathrm{jh}, \mathrm{ch}, \mathrm{sh}, \mathrm{r}$, an, en, ang, eng, ai, ei, ao, ou." And they perform better in single-spelling alphabet than in two-spelling or three-spelling. Among the new immigrant's children, the correctness they pronounce in vowels are " $\mathrm{b}$ ", the most accurate, then " $\mathrm{m}, \mathrm{p}, \mathrm{f}, \mathrm{d}$ ", and " $\mathrm{p}$ " is the worst one for them to know in vowels. On the other hand, "a"is the most accurate consonant for them, then following consonants "ao, i, u, o" while "er"becomes the hardest one. And in terms of tone, the forth sound is the sound with the most correctness then followed by the first and the third sound while the second sound is the one with the least correctness. From the other research we are convinced that the students who have barrier in distinguishing the sounds tend to confuse these highly similar sounds: the phonologically similar sounds, "ai, ei", "o, ou", "an, ang", "jh, z" , "ch, c", "sh, s", "ji, ci, si" morphologically similar sounds, "m,f”, "s, eng ”, “o, e", "h, ei” and both phonologically and morphologically similar sounds "eng, en."

\subsection{Special Cases}

There are also some cases that students might make a mistake like adding a interval sound, for example, they might pronounce "i, ê" when they are just try to pronounce "ê", "u, ei" for "ei", "tien" for "tan," "ling" for "leng" etc. Weak recognition in Chinese phonetic alphabets will lead to the learning difficulties in making words, making sentences, weak rhetoric skills, scribble, and incorrect characters. From the tablet of new immigrants' ability on Chinese phonetic alphabets, it shows that from the performance of general Chinese phonetic alphabets, there is no distinguish difference. But from the sub-exam, new immigrants' children normally have a comparatively bad performance on 
recognition and reading.

\section{Discussion}

The most important job of the elementary school is to teach children to read and write. The importance of teaching reading results from the fact that reading success is the key to educational success (Aaron, 1995; Hong, 1999; Burns, Griffin \& Snow; 1999). Hong (1999) stressed that access to further education, high-skilled jobs, and a chance to participate in the higher reaches of society depends in large part on school success, which itself is highly correlated with the ability to read. For students to succeed in reading, two components must be in place in our early childhood classrooms: a focus on phonetic notation in the Chinese language, similar to letters of the alphabet and systematic phonics instruction in the English language.

Our studies showed that local Taiwanese students were able to recognize and identify the names and the sounds of phonetic notation in Chinese before they even began formal schooling, and most students knew the entire set of phonetic notation by the middle of the first grade. Our studies report that students knew about half of them by the beginning of kindergarten and all of them by the end of kindergarten. They learned various skills such as visual discrimination of symbols, remembering names and sounds of phonetic notation, and coordinating visual and auditory relations.

However, results suggest that almost no South East Asian immigrant first grader were able to fully distinguish the sounds of different phonetic notations even by the middle of first grade. There is a distinct difference between the two groups' ability in the progress and effectiveness in learning phonetic notation in Chinese, as immigrant children cannot absorb or recognize as quickly as their local counterparts.

This may be linked to their family background, as well as education background of their family, where one parent, being the breadwinner of the family, is occupied with work and unable to tutor the child at home, and the other parent, usually the immigrant bride, is unable to assist the child in areas of Mandarin Chinese.

\section{References}

Aaron, P. G. (1995). Differential diagnosis of reading disabilities. School Psychology Review, 24(3), 345-356.

Bernstein, D., \& Tiegerman-Farber, E. (2002). Language and communication disorders in children (5th ed.). Needham Heights, MA: Allyn and Bacon.

Bernthal J. E., \& Bankson, W. B. (2004). Articulation and Phonological Disorders (5th Ed.). Allyn \& Bacon, Boston, MA.

Burns, M. S., Griffin, P., \& Snow, C. E. (Eds.). (1999). Starting out right: A guide to promoting children's reading success. Washington, DC: National Academy Press.

Chen, S.C. (2003). The Spread of English in Taiwan: Changing Uses and Shifting Attitudes. Taipei: Crane Publishing, Co.

Goodman, K. (1992). I don't found whole language. The Reading Teacher, 46(3), 188-199.

Ho, C. S. H., Chan, D. W., Chung, K. K., Lee, S. H., \& Tsang, S. M. (2007). In search of subtypes of Chinese developmental dyslexia. Journal of experimental child psychology, 97(1), 61-83. http://dx.doi.org/10.1016/j.jecp.2007.01.002

Hong, H. F. (1993). Orthography and reading disability -- A follow up study of Chinese reading disabled children. Taiwan, Chiayi: National Chung Cheng University, the Institute of Psychology Dissertation.

Hong, L. Y. (1999). A Comparison of Cognitive Components of Word Recognition of the Second Graders With and Without Word Recognition Difficulties. Taipei: Center of special education, National Taiwan Normal University.

Hu, Y. C. (2001). The Teaching Effects of Mandarin Phonetic Symbols learning's Related Factors and Meaningful Mandarin Phonetic Symbols of The Elementary School First-Grade Students with Reading difficulties. National Ping Tung University of Education Academic Journal, 15, 101-140.

Huang, X. S. (1997). Early Stages of Phonetic Recognition in Children and How It Affects Ability in Character Recognition Three Years Later. Tainan Teacher Training Institution, 5, 125-138.

Kuder, S. (1997). Teaching students with language and communication disabilities. Boston: Allyn \& Bacon. 
Lin, C. S. (1997). Educational psychology: recognition. Taipei: Yuan Liou publisher.

Ministry of Education Department of Statistics. (2005). A survey of foreign brides's elementary school children's learning and life intentation. Ministry of Education department of statistics. Taipei.

Pumfrey, P.D., \& Elliott, C.D. (Eds) (1990). Children's Difficulties in Reading, Spelling and Writing Challenges and Responses. London: Falmer Press.

Stanovich, K. E., \& Stanovich, P. J. (2006). Fostering the scientific study of reading instruction by example. In K. Dougherty Stahl \& M. McKenna (Eds.), Reading research at work: Foundations of effective practice (pp. 36-44). New York: Guilford Press.

Tan, G. D. (1998). The Research on Aboriginal Education. Taipei: Wu-Nan Library Publisher.

Zeng, R. Q. (2003). Promoting Reading Habits Amongst Learners In Imagination Development. Taiwan Education, 621, 50-55.

Zeng, S. J. (1996). Research Analyses of Reading Process For The Mentally Handicapped and Average Learners. Learning year of 1998 at Teacher Training Institution, Thesis Publication Ceremony (pp.17-30), Taipei: Department of Education.

Zhang, B. L. (1997). The research unhearing-impaired students' ability of obtaining notional phonetic alphabet. Bulletin of special education, 15, 151-172.

Zhong, R. F. (2004). Guideline of Hakanese in Taiwan. Taipei: Wu-Nan publisher. 